\title{
Cardio metabolic risk profile in women: an overview
}

\begin{abstract}
Cardio metabolic disorders traditionally perceived as male disease has now been recognised as a major health hazard affecting equally the women, especially with advancing age. However, in recent years, gender differences in prevalence of various cardio metabolic risk factors and their correlates have been documented from different corners of the world. Exploration of gender specific differences in cardio metabolic profile provides basis for formulating sex specific treatment strategies and management of patients with cardio metabolic disorders. Understanding physiological and psychosocial correlates of cardio metabolic disorders unique for women needs more attention for prevention and improvement in health care delivery to the affected population.
\end{abstract}

\author{
Volume 3 Issue I - 2016
}

Dipayan Choudhuri, Soma Choudhuri
Department of Human Physiology, Tripura University, Inda

Correspondence: Dipayan Choudhuri, Associate Professor Department of Human Physiology, Tripura University (A Central University), Suryamaninagar, Tripura - 799 022, Tel + 91 9436461010,Email dipayanchoudhuri@gmail.com

Received: September 20, 2016 | Published: November 10, 2016

\section{Introduction}

In recent years, cardio metabolic disorders like hypertension and type 2 diabetes mellitus have emerged as major health problem globally and one of the prime causes of death both in men and in women over 50years of age. The burden of cardio metabolic risk in young women is often misperceived owing to the fact that they are generally protected from such risk because of prominent presence of female sex hormone oestrogen during premenstrual period of their life. However, recent evidences indicated that mortality due to cardio metabolic diseases is increasing day by day in women between the ages of 35 and 54years. This may be explained by high incidence of obesity and overweight with accumulation of visceral fat and various hormonal dysfunction in this group of women that over rides the protective action of oestrogen..$^{1,2}$ Most of the traditional cardio metabolic risk factors are found to have differential impact on men and women. Total cholesterol (TC), triglyceride (TG), low-density lipoprotein (LDL-C) increases sharply in women after menopause. High density lipoprotein (HDL-C) levels are higher in women than in men from young adult hood onwards, which gradually declines after menopause. The loss of protection from HDL-C is considered to be a major cause behind increased cardio metabolic risk in postmenopausal women. Isolated low HDL-C represents a major cardio metabolic risk in women of all ages. Therefore, it is important to adopt a gender specific approach in interpreting HDL-C levels. Similarly elevated triglyceride possess a greater risk for cardio metabolic disorders in women than in men. ${ }^{3,4}$

Systolic hypertension is a common finding in women more than 55 years compared to men, and this may be related to decline in oestrogen levels during menopausal transition. Changes from a gynoid to an android pattern with onset of menopause results in increased visceral fat that act as a major cardio metabolic risk factor in presence of other comorbid conditions. Obesity is linked to increased blood sugar level, another major cardio metabolic risk factor in women. Women with diabetes are found to be more susceptible to cardio metabolic complications than their male counterparts. In addition, increased homocysteine, cigarette smoking and polycystic ovarian syndrome (PCOS) in women are found to promote cardio metabolic risk, lipoprotein (a) level is found to associated more with cardio metabolic risk in men than in women. ${ }^{4,5}$
Recent evidences indicated presence of higher levels of inflammatory markers and different biomarkers of oxidative stress in elderly women than in elderly men. In fact, elevation of oxidative stress levels, in recent times, represented the only strong independent risk indicator for cardio metabolic disorders in elderly women. While the molecular mechanisms for overwhelming gender disparity regarding oxidative stress indicators in cardio metabolic disorders is still debated, it is likely that they are related to hormonal status and are associated with loss of oestrogen dependent anti-oxidant effects in elderly women. Several inflammatory markers were evaluated during last two decades to ascertain their association with various cardio metabolic risk indicators in subjects. Of these markers, only high sensitive C-reactive protein (hs-CRP) was found to be clinically useful to predict cardio metabolic risk in both male and female subjects. In women, hs-CRP levels have been found to be highly predictive of future vascular disease and also to have implications for use of hormone replacement therapy (HRT). N-terminal pro-B type natriuretic peptide which has recently been established to have strong relationship with incident cardiovascular disease have found to have strong predictive value in women than men.4-6

The recognition of sex differences, in the pathophysiology of cardio metabolic disorders had lead to more women specific studies on cardio metabolic risk factors and their correlates. However, the number of identified variables contributing differentially in women and men are quite large and are growing day by day. In addition to the traditional risk factors various noble factors have now been emerged as potential risk factors for cardio metabolic diseases and responsible for gender disparity in expression of traditional risk factors. These noble factors include sex chromosome, hormonal status, disorders related to reproduction and pregnancy, aging and gender specific psychological and psychosocial variables. It is, therefore, important to include these variables in future study design on cardio metabolic risk assessment to obtain sex specific data on these variables. Moreover, sex specific reference values for various cardiac biomarkers are yet to be established. The failure to establish sex specific, clinically applicable reference values for cardiac biomarkers may contribute to under diagnosis and elevated death rate from cardio metabolic disorders in women. ${ }^{7}$

\section{Acknowledgements}

None. 


\section{Conflict of interest}

The author declares no conflict of interest.

\section{References}

1. Saha T, Palaskas VN, Ahmed A. An update on gender disparities in coronary heart disease care. Curr Atheroscler Rep. 2016;18(5):28.

2. Del Principe $\mathrm{D}$, Ruggieri $\mathrm{A}$, Pietraforte $\mathrm{D}$, et al. Int $J$ Cardiol. 2015;187:291-298.

3. Rochlani Y, Pothineni NV, Mehta JL. Metabolic syndrome: does it differ between women and men? Cardiovasc Drugs Ther. 2015;29(4):329338.
4. Vassalle C, Mercuri A, Maffei S. Oxidative status and cardiovascular risk in women: keeping pink at heart. World J Cardiol. 2009;1(1):26-30.

5. Srivastava AQ, Singh HV, Raizada A, et al. C-reactive protein, inflammation and coronary heart disease. The Egyptian Heart Journal. 2015;67(2):89-97.

6. Everett BM, Berger JS, Manson JE, et al. B-type natriruetic peptides improve cardiovascular disease predictionin cohort of women. J Am Coll Cardiol. 2014;64(7):1778-1797.

7. Sanghavi M, Gulati M. Sex difference in pathophysiology, treatment and outcome in IHD. Curr Atheroscler Rep. 2015;17(6):511. 